\title{
Marcas como um indicador: revisão sistemática e análise bibliométrica da literatura
}

\author{
Adonis Reis de Medeiros Fillho \\ Suzana Leitão Russo \\ Universidade Federal de Sergipe - UFS, Brasil
}

ORIG INAL

\begin{abstract}
Resumo
Objetivo. O estudo objetiva investigar, apresentar e discutir os conceitos, contextos e aplicações referentes ao uso de marcas como indicadores, revelando o que pode ser entendido a partir de pedidos de registro de marcas, e quais conclusões podem ser inferidas com fundamento nas publicações encontradas.

Método. Para satisfazer os objetivos, técnicas de revisão sistemática da literatura, análises bibliométrica e sistêmica foram empregadas. Utilizando-se os programas Microsoft Excel e VOSviewer, foram feitas avaliações de publicações por ano, fator de impacto dos periódicos, citações e análises network de coautoria, citações, filiação, termos utilizados e cocitação de referências.

Resultados. A análise biliométrica encontrou pouca quantidade de publicações, uma tendência a não evolução no número anual de publicações e a presença de publicações carentes de avaliação editorial. Ao passo que outros documentos foram publicados em periódicos com alto fator de impacto e possuem notável incidência de citações. A análise sistêmica mostrou ampla gama de possibilidades do uso de marcas como indicadores, como indicador de novas atividades econômicas, para prever mudanças econômicas, explorar sistemas de inovação regionais, indicador de competitividade internacional, porém, especialmente para seu uso como indicador de inovação, o que ocorreu com maior frequência.

Conclusões. Diante de tantas possibilidades relatadas ressalta-se a importância dos, ainda não tão explorados, dados de marcas para os mais variados usos. Particularmente quando relacionadas inovação e serviços, tendo em vista o fenômeno da servitização das economias. Bem como a necessidade de avanço e aprofundamento das pesquisas e seu potencial de impacto, revelado pelas publicações já existentes.
\end{abstract}

Palavras-chave

Bibliometria; Econômico; Indicador; Inovação; Marcas; Revisão sistemática

\section{Trademarks as an indicator: systematic review and bibliometric analysis of literature}

\begin{abstract}
Objective. The study aims to investigate, present and discuss the concepts, contexts and applications regarding the use of trademarks as indicators, revealing what can be understood from trademark applications and which conclusions can be inferred based on the publications found.

Method. To meet the objectives, techniques of systematic review, bibliometric and systemic analyzes were employed. Using the Microsoft Excel and VOSviewer programs, evaluations of publications per year, periodic impact factor, citations, and network analyzes about co-authorship, citations, filiation, terms used and cocitation of references were made.

Results. The biliometric analysis found a low number of publications, a trend of non-evolution in the annual number of publications and the presence of publications lacking editorial evaluation. While other documents have been published in journals with high impact factor and have a notable incidence of citations. The systemic analysis showed a wide range of possibilities for the use of brands as indicators, as indicators of new economic activities, to predict economic changes, to explore regional innovation systems, an indicator of international competitiveness, but especially for its use as an indicator of innovation. Which occurred more frequently.

Conclusions. Faced with so many possibilities, it is important to note the importance the not yet explored adoption of trademarks for the most varied purposes. Particularly when related to innovation and services, in view of the phenomenon of the servitization of economies. As well as the need to advance and deepen the research and its potential impact, revealed by the existing publications.
\end{abstract}

Keywords

Bibliometric study; Economic; Indicator; Innovation; Systematic review; Trademarks 


\section{Introdução}

De acordo com WTO (1994) "Qualquer sinal, ou combinação de sinais, susceptível de distinguir produtos ou serviços de uma empresa dos de outras empresas, deve ser capaz de constituir uma marca". Tal definição abrange dois aspectos, o de distinguir os bens e serviços de uma determinada empresa daqueles de outras empresas e, ao mesmo tempo, individualizar bens e serviços para o consumidor, identificando a sua fonte (WIPO, 2008). Historicamente, o uso de marcas refere-se ao início da humanidade (ONO, 1999; WIPO, 2008) e, atualmente, são atribuídas diferentes funções às marcas, além de seu papel primário de distinguir bens e serviços.

É necessário reconhecer e proteger as marcas em mercados competitivos dada a sua função econômica de reduzir os custos de transação, minimizando o esforço e o tempo gasto pelos consumidores na distinção do que é oferecido. Tais reduções nos custos de transação ocorrem devido às funções de fonte e confiança exercidas pela marca, que liga o produto ou serviço ao seu titular e confere aos consumidores a confiança de que o proprietário da marca exerce controle na fabricação do produto ou na prestação do serviço (ALBUQUERQUE, 2015).

A função econômica é observada, como um redutor de custo transacional, quando um novo produto ou serviço é lançado e ostenta a marca de uma empresa de renome, mesmo se essa fama é o resultado do desempenho da empresa em outra área (MARQUES; MAGALHÃES, 2009). O que demonstra o importante papel estratégico desempenhado pelas marcas como fator contribuinte para o progresso econômico (MARQUES; MAGALHÃES, 2009; WIPO, 2008).

O registro de uma marca assegura direitos exclusivos e permite ao detentor investir no posicionamento e desenvolvimento de seus bens e serviços, garantindo os benefícios de internalização desses investimentos (GARCÍA, 2015). A proteção, decorrente do registo, tem um duplo objetivo, evitar confusão entre os consumidores sobre a origem de um bem ou serviço oferecido no mercado e proteger a reputação do comerciante. Ao garantir aos consumidores a identificação precisa da origem dos produtos que compram, a lei preserva, consequentemente, a integridade do mercado (BROWN; NAGY, 2015). Isso ocorre pois, ao ser utilizada ao mesmo tempo por pessoas diferentes, a marca pode ter o seu carácter distintivo diluído, perdendo a capacidade de atestar aos consumidores que todos os bens ou serviços provêm de uma única origem comercial (PLAZA, 2015).

Ademais, as marcas são uma parte importante no processo de comercialização de uma invenção, sendo um indicador potencial de inovação de produtos e mudança setorial (MENDONÇA; PEREIRA; GODINHO, 2004). E pode trazer outra perspectiva sobre o processo inovativo: o aspecto comercial das inovações, que os dados de Pesquisa e Desenvolvimento (P\&D) e patentes não podem fornecer (MILLOT, 2009).

Os pedidos de registro de marcas podem indicar, ainda, a possibilidade de lançamento de novos produtos no mercado, uma vez que é provável que as empresas registrem marcas para produtos que são resultados de investimentos em P\&D como forma de estender a proteção das inovações (MALMBERG, 2005).

Vários estudos têm demonstrado, através da análise empírica de dados, que as marcas possuem uma correlação com atividades inovadoras, uma vez que estão ligadas à comercialização de bens ou serviços, enquanto os dados de P\&D não refletem o sucesso técnico ou comercial de pesquisas, e dados de patentes muitas vezes mostram invenções que não geram aplicações úteis e não são comercializadas (MILLOT, 2009). Além disso, dados de marcas representam, ainda, uma vantagem em relação às patentes por serem menos dependente das empresas de atividade tecnológica (MENDONÇA et al., 2004). Logo, qualquer empresa, ao utilizar o registro de marca, está apta a fornecer informações sobre qualquer ramo de atividade.

Consequentemente, tais dados, são uma fonte de informação quantitativa e qualitativa sobre a atividade socioeconômica (MENDONÇA et al., 2004), uma vez que também estão relacionados a inovações não tecnológicas, que se tornaram cada vez mais importantes em economias baseadas em serviços (MILLOT, 2009). 
Utilizando métodos de revisão sistemática e de bibliometria, para coletar e analisar dados de diferentes bases de dados, o estudo tem por objetivo investigar, apresentar e discutir os conceitos, contextos e aplicações referentes ao uso de marcas como indicadores, revelando o que pode ser compreendido a partir dos seus dados, e quais conclusões podem ser inferidas com fundamento nas publicações encontradas.

Apesar de a importância que as marcas possuem atualmente, tanto para as empresas quanto no cenário econômico mundial, ser praticamente senso comum entre todos os que estudam as marcas sob as mais diversas perspectivas, pouco se conhece acerca de aplicações empíricas ou interpretações possíveis a respeito dos seus dados. E é nessa conjuntura que esta pesquisa encontra razão e se mostra significativa. Espera-se, como consequência, encontrar estudos que norteiem e demonstrem as possibilidades de aplicação de dados de marca e quais possíveis conclusões pode-se chegar fundamentado em sua análise.

\section{Metodologia}

Seguindo as classificações propostas por Prodanov e Freitas (2013), este trabalho é classificado, do ponto de vista da sua natureza, como uma pesquisa básica, com a intenção de gerar conhecimento para o progresso da ciência sem uma aplicação prática planejada. No que se refere aos seus objetivos é exploratório-descritivo, exploratório por fornecer mais informações, orientar a determinação de objetivos e a formulação de hipóteses ou descobrir um novo tipo de abordagem ao assunto, e descritivo por tentar descobrir com que frequência ocorre um evento, sua natureza, suas características, causas e relações com outros fatos. Concomitantemente, quanto aos procedimentos técnicos, é qualificado como bibliográfico por ser elaborado a partir de fontes bibliográficas publicadas.

Com o intuito de satisfazer os objetivos propostos, os dados foram coletados utilizando-se de técnicas de revisão sistemática da literatura, um método que tenta coletar todas as evidências empíricas de acordo com critérios de elegibilidade estabelecidos, com o objetivo de responder a uma pergunta de pesquisa específica (HIGGINS; GREEN, 2011). É um meio de contribuir sobre o que funciona e o que não funciona, e identifica onde pouca ou nenhuma pesquisa relevante foi feita e onde novos estudos são necessários (PETTICREW; ROBERTS, 2008).

Tal método sintetiza todas as evidências de alta qualidade disponíveis, fornecendo uma base de evidência forte para orientar a política e a prática (VICTOR, 2008), geralmente utilizado quando um cenário preciso de pesquisas anteriores é essencial para promover o desenvolvimento de novas pesquisas (PETTICREW; ROBERTS, 2008), ou quando existe a necessidade de resumir todas as informações atuais sobre algum fenômeno de forma completa e imparcial (KITCHENHAM, 2004).

Conjuntamente, foi feita uma análise bibliométrica para explorar e avaliar os dados coletados na revisão sistemática. O princípio da bibliometria é analisar a atividade científica ou técnica por meio de estudos quantitativos de publicações, além disso, os indicadores quantitativos, além de apoiar a compreensão da dinâmica da ciência e da tecnologia, também funcionam como ferramentas para o planejamento de políticas e tomada de decisões neste setor (SANTOS, 2003).

Brereton et al. (2007) agruparam as etapas de uma revisão sistemática em três fases principais: planejamento, realização da revisão e relato da revisão. Incluindo-se aqui as principais características de uma revisão sistemática, conforme listado por Higgins and Green (2011):

- um conjunto claramente definido de objetivos com critérios de elegibilidade prédefinidos para os estudos;

- uma metodologia explícita e reprodutível;

- uma busca sistemática que tente identificar todos os estudos que atendam aos critérios de elegibilidade; 
- uma avaliação da validade dos resultados dos estudos incluídos, por exemplo através da avaliação do risco de viés; e

- uma apresentação sistemática e síntese das características e resultados dos estudos incluídos.

Os documentos encontrados na pesquisa foram comparados com os critérios de inclusão previamente definidos para determinar a relevância do estudo, quais sejam: (i) Publicado até dezembro de 2016; (ii) Publicado em inglês, espanhol ou português; (iii) Relacionando as marcas com qualquer tipo de indicadores; e (vi) Possuir resumo e texto completo disponíveis. Ante a variedade de publicações encontradas, todos os tipos de publicações foram considerados para as análises e, para efeito de equiparação, todas serão chamadas de "documentos".

A coleta de dados foi realizada nos seguintes bancos de dados: ScienceDirect, Scopus, Web of Science, Emerald Insight, Wiley Online Library, IEEE Xplore Digital Library e ProQuest, fazendo uso da opção mais ampla disponível em cada base de dados ${ }^{1}$. A estratégia de pesquisa foi realizada no campo "Título"2 e utilizou-se as palavras-chave combinadas com os operadores booleanos, conforme descrito na Tabela 1.

Tabela 1. Estratégia de pesquisa por base, idioma e respectivos resultados.

\begin{tabular}{|c|c|c|c|c|c|c|c|}
\hline \multirow[b]{2}{*}{ Combinação/Língua } & \multicolumn{7}{|c|}{ Base de dados } \\
\hline & $\begin{array}{c}\text { Science } \\
\text { Direct }\end{array}$ & Scopus & $\begin{array}{l}\text { Web of } \\
\text { Science }\end{array}$ & Emerald & Wiley & $\begin{array}{l}\text { IEEE } \\
\text { Xplore }\end{array}$ & ProQuest \\
\hline \multicolumn{8}{|l|}{ Inglês } \\
\hline $\begin{array}{c}\text { Trademark OR Mark } \\
\text { OR Brand AND } \\
\text { Indicator }\end{array}$ & 19 & 29 & 1.303 & 1 & 6 & 30 & 34 \\
\hline $\begin{array}{c}\text { Trademark OR Mark } \\
\text { OR Brand AND } \\
\text { Pointer }\end{array}$ & 0 & 5 & 508 & 4 & 0 & 14 & 10 \\
\hline $\begin{array}{c}\text { Trademark OR Mark } \\
\text { OR Brand AND } \\
\text { Index }\end{array}$ & 102 & 111 & 1.245 & 8 & 27 & 133 & 69 \\
\hline $\begin{array}{c}\text { Trademark OR Mark } \\
\text { OR Brand AND } \\
\text { Gauge }\end{array}$ & 1 & 6 & 581 & 1 & 0 & 13 & 13 \\
\hline
\end{tabular}

Espanhol

\begin{tabular}{l|l|l|l|l|l|l|l}
\hline $\begin{array}{c}\text { Marca AND } \\
\text { Indicador }\end{array}$ & 3 & 0 & 0 & 0 & 0 & 0 & 0 \\
\hline
\end{tabular}

\section{Português}

\begin{tabular}{c|c|c|c|c|c|c|c}
\hline $\begin{array}{c}\text { Marca AND } \\
\text { Indicador }\end{array}$ & 3 & 0 & 0 & 0 & 0 & 0 & 0 \\
\hline Total por Base & 128 & 151 & 3.637 & 14 & 33 & 190 & 126 \\
\hline Total Geral & & & & & & & 4.279 \\
\hline
\end{tabular}

Fonte: Elaboração Própria 
Cada autor fez as avaliações de maneira independente, analisando títulos, resumos e textos completos das publicações identificadas que preencheram os critérios de inclusão. A inclusão de documentos no estudo baseou-se na concordância entre os dois revisores. Em caso de desacordo, o documento foi considerado elegível para a etapa subsequente até que houvesse consenso sobre sua permanência.

Os resultados foram analisados utilizando-se os programas Microsoft Excel e VOSviewer. Foram feitas análises tais como: publicações por ano, fator de impacto dos periódicos, quantidade de citações, análise sistêmica dos documentos, e análises de rede (network) de coautoria, citações, organizações as quais os autores são filiados, termos utilizados e cocitação de referências.

\section{Apresentação e análise dos resultados}

Nesta seção, os resultados da pesquisa serão delineados, de acordo com os dados dos pesquisadores e divididos por tipo de análise realizada: bibliométrica, de rede (network) e sistêmica, respectivamente.

\subsection{Análise bibliométrica}

A pesquisa inicial obteve 4279 documentos, dos quais 363 estavam em duplicidade e foram excluídos. Os 3916 títulos foram analisados, sendo selecionados e avaliados 34 resumos potencialmente relevantes. E um total de 10 documentos foram completamente lidos, dos quais 3 (três) foram excluídos por não atenderem aos critérios de inclusão e 2 (dois) adicionados de suas referências, totalizando 9 documentos selecionados por satisfazerem os critérios de elegibilidade, conforme demonstrado na Figura 1.

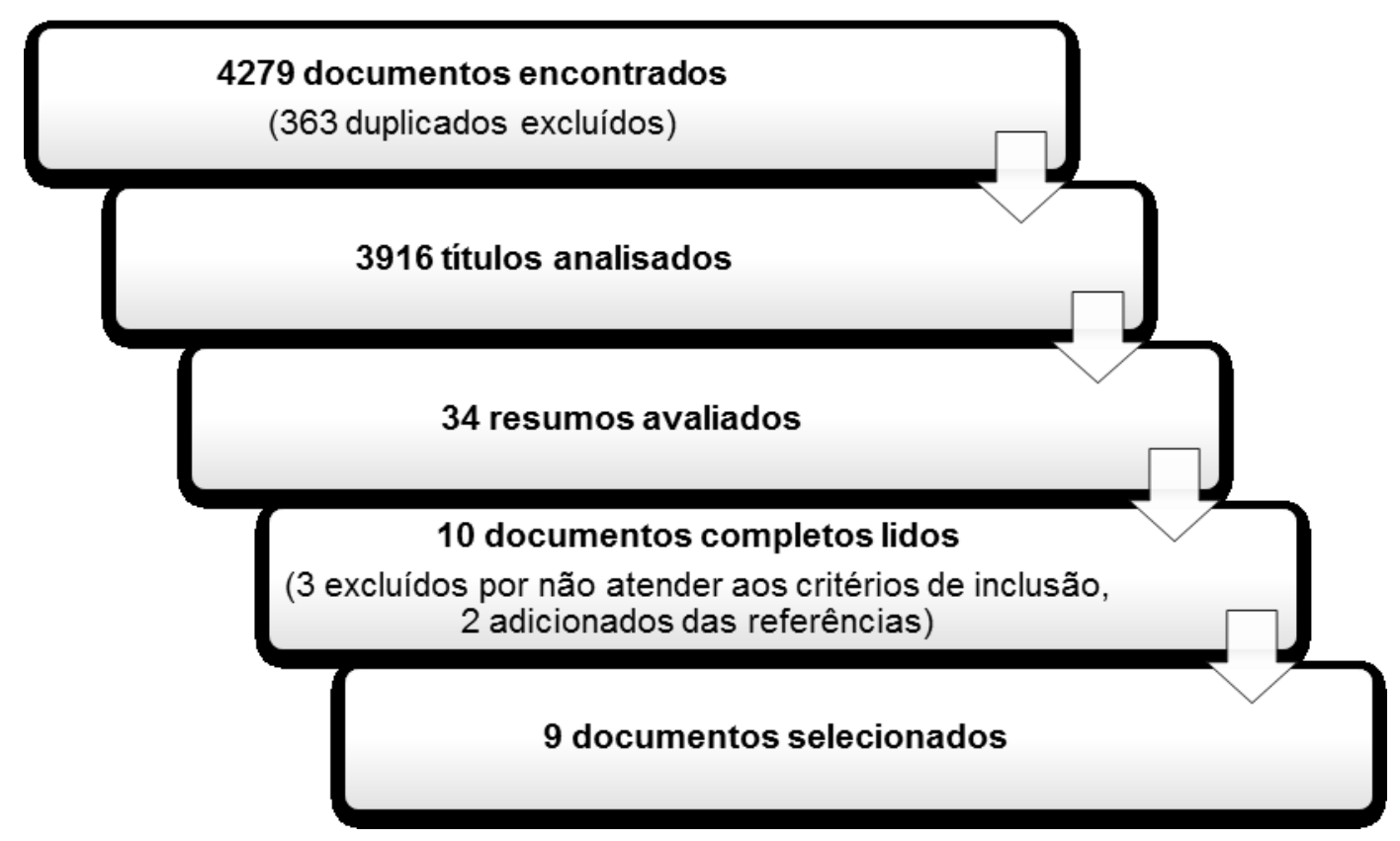

Figura 1. Fluxograma do estudo.

Após as buscas e análises completas os nove documentos selecionados para compor o estudo foram relacionados e são apresentados, por ordem alfabética, na Tabela 2. Sendo cinco artigos científicos publicados em periódicos, dois capítulos de livros e dois Working Papers ${ }^{3}$.

A Figura 2 traz o ano de publicação dos documentos selecionados, mostrando que as publicações ocorreram durante o período de 2000 a 2014, com poucas publicações por ano e sem uma regularidade anual bem definida. A linha de tendência da figura aponta para a permanência das publicações na ordem de um, com uma 
leve inclinação para baixo nos anos subsequentes, ou seja, não haverá evolução no número de publicações ou que as mesmas vão ocorrer com menor frequência anual.

A avaliação dos periódicos que publicaram os documentos selecionados foi levantada a partir dos dados mais recentes disponíveis dos índices de fator de impacto Journal Citation Reports (JCR) e Scientific Journal Rankings (SJR), índice h e índice de imediação. Os resultados são apresentados na Tabela 3. Como nem todos os documentos são oriundos de periódicos, não foi possível avaliar toda a amostra, entretanto dos nove documentos foi possível avaliar os periódicos de seis.

Tabela 1. Referências completas dos documentos selecionados.

\begin{tabular}{|c|}
\hline pletas \\
\hline $\begin{array}{l}\text { 1. } \text { FLIKKEMA, Meindert; DE MAN, Ard-Pieter; CASTALDI, Carolina. Are Trademark Counts a Valid Indicator of } \\
\text { Innovation? Results of an In-Depth Study of New Benelux Trademarks Filed by SMEs. Industry and Innovation v. 21, } \\
\text { n. 4, p. 310-331, 2014. }\end{array}$ \\
\hline $\begin{array}{l}\text { 2. GATRELL, Jay D; CEH, S L Brian. Trademark Data as Economic Indicator: The United States, 1996-2000. } \\
\text { The Great Lakes Geographer v. 10, n. 1, p. 46-56, } 2003 .\end{array}$ \\
\hline $\begin{array}{l}\text { 3. MALMBERG, Claes. Trademarks statistics as innovation indicator? - A micro study. CIRCLE Electronic } \\
\text { Working Paper Series, No. 17, vol. 2005, CIRCLE, Lund University, Lund, } 2005 .\end{array}$ \\
\hline $\begin{array}{l}\text { 4. MEEUSEN, Wim; RAYP, Glenn. Patents and trademarks as indicators of international competitiveness: the } \\
\text { VAR versus the hysteresis approach. In P. Buigues (Ed.), Competitiveness and the value of intangible assets. } \\
\text { Cheltenham: Edward Elgar, 2000. p. } 273-296 \text {. }\end{array}$ \\
\hline $\begin{array}{l}\text { 5. MENDONÇA, Sandro. Trademarks as a telecommunications indicator for industrial analysis and policy. In: } \\
\text { Telecommunication Economics. Springer Berlin Heidelberg, 2012. p. 33-41. }\end{array}$ \\
\hline $\begin{array}{l}\text { 6. MENDONÇA, Sandro; PEREIRA, Tiago Santos; GODINHO, Manuel Mira. Trademarks as an indicator of } \\
\text { innovation and industrial change. Research Policy, v. 33, n. 9, p. 1385-1404, } 2004 .\end{array}$ \\
\hline $\begin{array}{l}\text { 7. MILLOT. Valentine. Trademarks as an Indicator of Product and Marketing Innovations. OECD Science, } \\
\text { Technology and Industry Working Papers, No. 2009/06, OECD Publishing, Paris, } 2009 .\end{array}$ \\
\hline SCHMOCH, Ulrich. Service marks as novel innovation indicator. Research Evaluation v. 12, n. 2, p. 149-156, \\
\hline $\begin{array}{l}\text { ce marks as indicators for innovation in knowledge-based } \\
009 \text {. }\end{array}$ \\
\hline
\end{tabular}

Fonte: Elaboração Própria

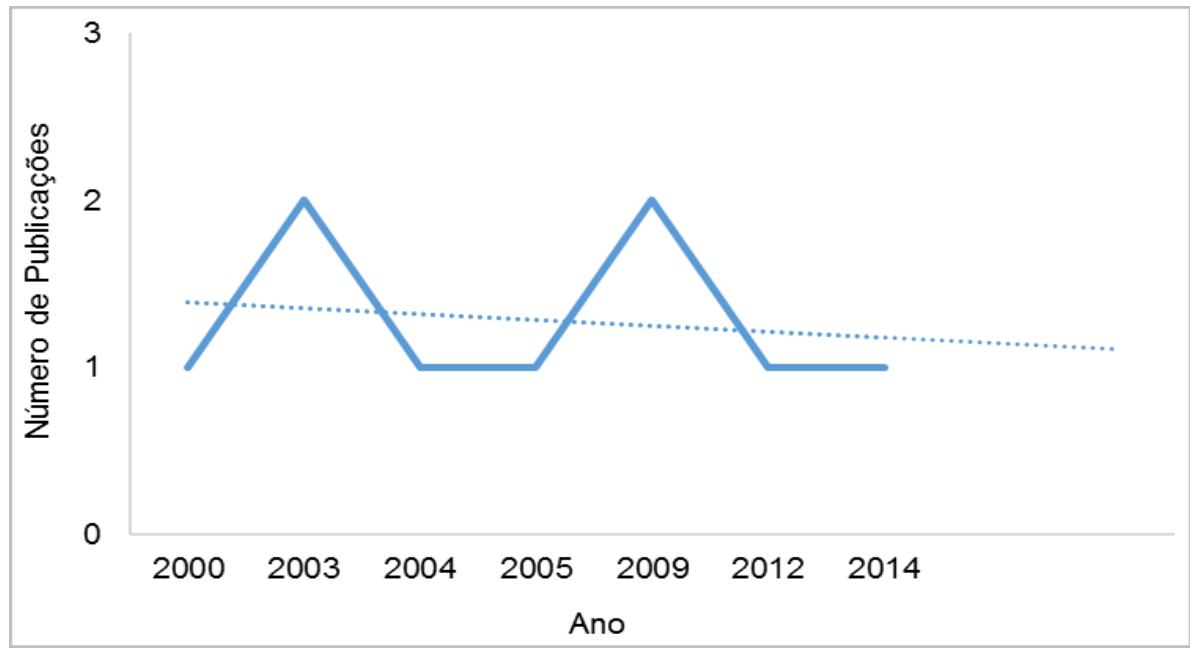

Figura 1. Ano de publicação dos documentos.

Apesar de ainda criticado, o fator de impacto é considerado o melhor instrumento de avaliação de qualidade de revistas científicas. O fator de impacto JCR possui uma vantagem sobre o SJR a ser levada em consideração que é sua tradição. Contudo, estudos demonstraram correlação moderadamente forte entre os índices, apesar 
de diferenças significativas em alguns campos científicos, especialmente da área médica (FALAGAS et al., 2008).

No entanto, a escolha de um índice em detrimento do outro é uma questão metodológica, que envolve saber se qualidade ou popularidade é considerado critério principal para a avaliação de periódicos. As principais diferenças entre o JCR e o SJR decorrem especialmente das diferentes bases de dados utilizadas como fontes de citações e de diferenças na estimação destes índices (FALAGAS et al., 2008).

Tabela 2. Avaliação dos periódicos.

\begin{tabular}{l|c|c|c|c}
\multicolumn{1}{c|}{ Título da Publicação } & $\begin{array}{c}\text { Fator de } \\
\text { impacto } \\
\text { JCR }^{1}\end{array}$ & $\begin{array}{c}\text { Fator de } \\
\text { impacto } \\
\text { SJR }\end{array}$ & h-index & $\begin{array}{c}\text { Índice de } \\
\text { Imediação }^{2}\end{array}$ \\
\hline Great Lakes Geographer & N.D. & $0,122^{a}$ & 7 & N.D. \\
\hline Industry and Innovation & $0,870^{\text {b }}$ & $1,298^{\text {b }}$ & 41 & 0,033 \\
\hline Lecture Notes in Computer Science & $0,402^{c}$ & $0,252^{\text {b }}$ & 177 & 0,402 \\
\hline Research Evaluation & $1,467^{\text {b }}$ & $0,919^{\text {b }}$ & 29 & 0,143 \\
\hline Research Policy & $3,470^{\text {b }}$ & $3,536^{\text {b }}$ & 160 & 0,525 \\
\hline
\end{tabular}

${ }^{1}$ Fonte: SClmago Journal Rank;

${ }^{2}$ Fonte: Thompson Scientific Journal Citation Reports;

a Dado referente a 2013;

b Dado referente a 2015;

c Dado referente a 2005.

Fonte: Elaboração Própria

Os resultados apresentados na Tabela 3 apontam uma variedade na qualidade dos periódicos medida pelo fator de impacto, mas deixa claro a relevância e o potencial do estudo das marcas como um indicador, uma vez que, artigos foram publicados em revistas renomadas e com fator de impacto significante, especialmente para a área interdisciplinar, destacando-se entre elas a Industry and Innovation, Research Evaluation e Research Policy.

O h-index é um complemento útil para fatores de impacto de revistas, é robusto e combina o número de publicações (quantidade) e a taxa de citação (qualidade) de forma equilibrada, sendo calculado para o período de um ano (BRAUN; GLÄNZEL; SCHUBERT, 2006). Um h-index de 40 significa, por exemplo, que 40 artigos foram publicados e que cada um conteve pelo menos 40 citações (BORNMANN; DANIEL, 2007). Assim, destacaram-se os periódicos Lecture Notes in Computer Science e Research Policy com um h-index de 177 e 160 , respectivamente.

Em relação ao índice de imediação, que reflete a frequência em que a revista é citada por ano, a partir do cálculo do número médio de vezes que um artigo de um ano em uma revista específica é citado por outros artigos nesse mesmo ano (HUANG; CATHY LIN, 2012), neste caso os resultados exibiram números mais baixos, com destaque positivo para a revista Research Policy.

Foi realizada, também, uma avaliação da quantidade de citações que cada documento possui e um comparativo entre os dados da base de origem do documento e do Google Acadêmico, que podem ser vistos na Tabela 4.

Com exceção de um, os documentos apresentaram números maiores de citações quando consultados através do Google Acadêmico, motivo pelo qual optou-se por trabalhar com esta ferramenta para fins de quantidade de citações de cada documento.

Os dois documentos com maior número de citações, Mendonça et al. (2004) com 232 citações e Schmoch (2003) com 103 citações, são oriundos dos periódicos com maior fator de impacto JCR, Research Policy e Research Evaluation, respectivamente. E os dois seguintes, Millot (2009) e Malmberg (2005), são Working Papers, o que demonstra a importância desses tipos de publicação, ainda que em caráter inicial ou carentes de avaliação editorial, podem conter contribuições valiosas para o desenvolvimento de certos assuntos ainda pouco abordados. 
Tabela 4. Citações.

\begin{tabular}{l|c|c}
\multicolumn{1}{c|}{ Referência } & $\begin{array}{c}\text { Citações: } \\
\text { base de } \\
\text { origem }\end{array}$ & $\begin{array}{c}\text { Citações: } \\
\text { Google } \\
\text { Acadêmico }\end{array}$ \\
\hline Mendonça et al. (2004) & 74 & 232 \\
\hline Schmoch (2003) & 20 & 103 \\
\hline Millot (2009) & N.D. & 63 \\
\hline Malmberg (2005) & 11 & 35 \\
\hline Schmoch and Gauch (2009) & 8 & 28 \\
\hline Meeusen and Rayp (2000) & 0 & 12 \\
\hline Gatrell and Ceh (2003) & 2 & 10 \\
\hline Flikkema et al. (2014) & 1 & 8 \\
\hline Mendonça (2012) & 1 & 0 \\
\hline
\end{tabular}

Fonte: Elaboração Própria

\subsection{Análise de rede (network)}

A análise de rede, ou network, realizada na pesquisa foi feita utilizando-se o software VOSviewer, baseando-se no mapeamento bibliométrico dos dados auferidos nas bases consultadas.

Tal ferramenta presta especial atenção à representação gráfica de mapas bibliométricos, que é um importante tópico de pesquisa no campo da bibliometria. O VOSviewer pode ser usado, por exemplo, para construir mapas de autores ou revistas com base em dados de cocitações ou para construir mapas de palavras-chave com base em dados de coocorrência. O programa oferece, ainda, um visualizador que permite que mapas bibliométricos sejam examinados em detalhes. O software usa a técnica de mapeamento VOS, onde VOS significa visualização de similaridades (visualization of similarities), e na construção de mapas baseados em distância, que são mapas em que a distância entre dois itens reflete a força da relação entre eles. Uma distância menor geralmente indica uma relação mais forte (VAN ECK; WALTMAN, 2010).

A Figura 3 faz análise de rede em relação a coautoria entre todos os 14 autores referentes aos nove documentos. Cada círculo representa um autor e as linhas ligam os autores de um mesmo documento entre si, cada cor atribuída a um grupo significa os autores compartilham autoria de algum documento. Assim, pode-se observar que, embora o número de documentos seja pequeno há pouca interação entre eles no desenvolvimento das pesquisas.

Outro ponto mostrado na Figura 3 é a relação entre os autores, representada pela distância entre os grupos. Quanto mais próximos estão localizados, mais forte é sua ligação em termos de coautoria, assim observa-se pouca proximidade entre os grupos. Além disso, o tamanho de cada círculo retrata o número de citações de cada autor, logo quanto maior o círculo, mais citações o autor possui em relação ao documento selecionado, onde destacaram-se MENDONÇA, S. e GODINHO, M. M.

No tocante à relação entre citação e ano de publicação, a Figura 4 mostra que ela não existe nos documentos selecionados. Dentre os 14 autores, os mais citados não foram os pioneiros nem os que publicaram mais recentemente, mas os autores cuja publicação deu-se em periódicos com maior fator de impacto.

Em relação as organizações as quais os autores são filiados, a análise feita na Figura 5 foi análogo ao apresentado na Figura 3. A diferença se dá pois no cenário observado não há linhas interligando as instituições e o tamanho dos círculos é exatamente igual. Assim, demonstra ainda nenhuma interação, uma vez que as instituições aparecem apenas uma vez e sem ligação alguma entre si. 
ceh s.ı. b.

\section{mendonça s.}

godinhom. m.

meeusen w.

rayp g.

\section{flikkema m.}

castaldi c.

schmoch u.

malmberg c.

millot v.

Figura 2. Análise de coautoria.

ceh s.ı. b.

mendonça s.

godinho m. m.

\section{meeusen w. \\ raypg.}

flikkema $m$.

castaldi c. schmoch u.

malmberg c.

$2005 \quad 2010 \quad 2015$

Figura 3. Documentos mais citados por ano de publicação.

Entretanto, há de se observar que, como os dados trabalhados são retirados das informações bibliográficas dos documentos, estes contêm limitações importantes de se mencionar, especialmente a questão temporal da informação. O momento no qual a informação foi prestada difere para cada documento, pois se deu momentos antes da publicação. Pesquisadores podem mudar de departamento ou instituição, bem como estes podem ter 
suas designações alteradas e como isso não é captado pelo software, tal análise feita na Figura 5 pode não refletir completamente a realidade.

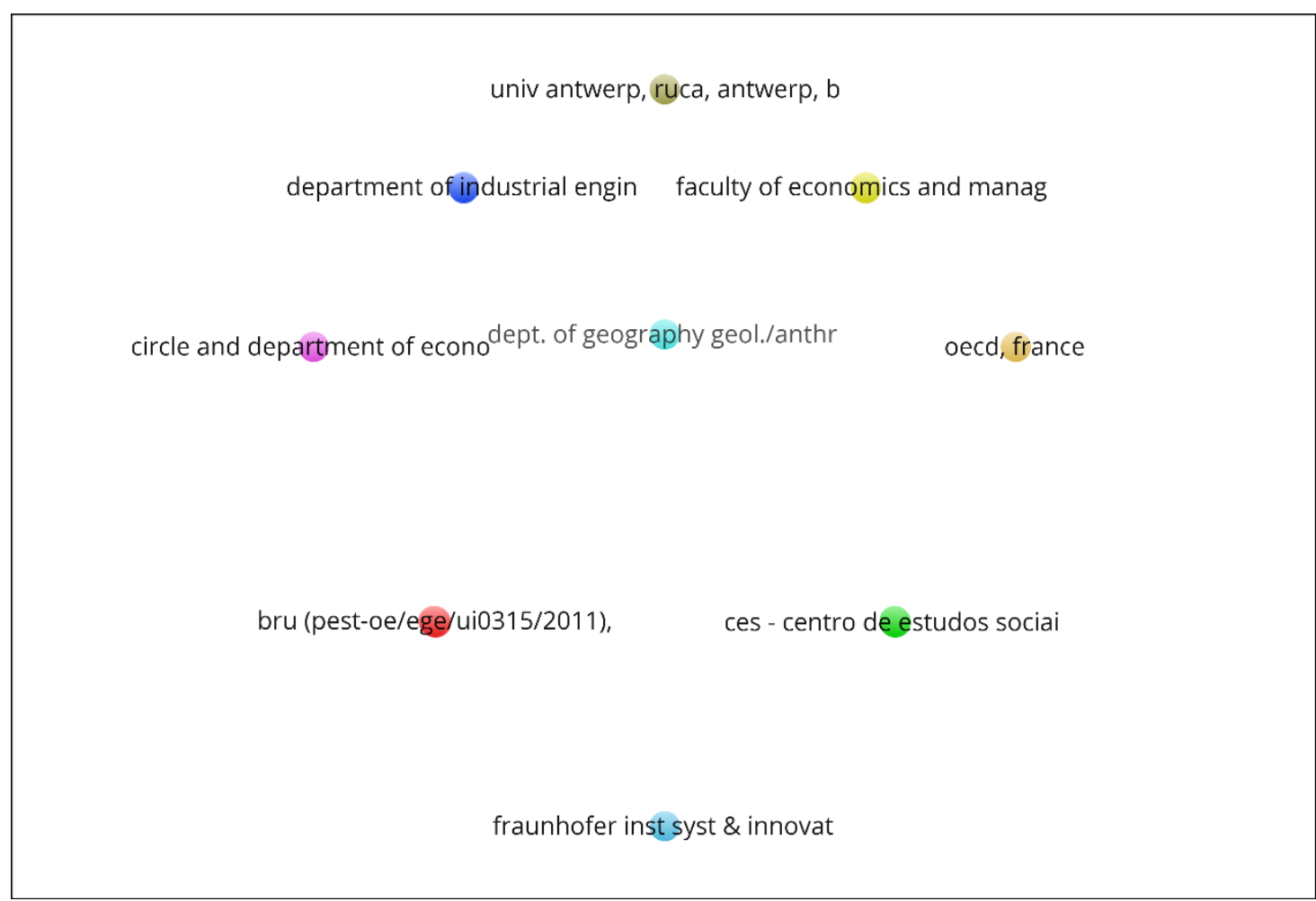

Figura 5. Organizações por documentos publicados.

Em relação aos termos utilizados nos títulos e resumos dos documentos, os que exibiram mais de uma ocorrência foram selecionados e uma avaliação de rede foi executada e apresentada na Figura 6. Do total de 240 termos encontrados, 45 foram mencionados mais de uma vez e compõem a rede.

Observa-se o termo trademark com centro da imagem com apresentando o maior círculo e sendo o núcleo de todas as ligações e muito próximo ao termo indicator. Como ambos os termos foram escolhidos como critérios de inclusão por ocasião da seleção dos documentos é natural que aparecem em destaque nesta análise. No tocante aos demais termos que se destacaram podemos ressaltar a presença dos termos innovation, product, service, trademark dada, innovation indicator e economic indicator.

Ademais, a análise reuniu os termos em três principais grupos identificados pelas cores azul, vermelho e verde. O grupo de cor azul é representado principalmente pelo termo trademark, o grupo vermelho é formado pelos termos, indicator, product, innovation indicator, service e service mark, e o grupo de cor verde é composto pelos termos innovation, patent, paper, trademark data e economic indicator.

Tal conjuntura conduz a interpretação de que a distância entre os termos que relacionam as marcas efetivamente como um indicador ainda demonstra um distanciamento, que tende a ser reflexo da pouca quantidade de estudos publicados sobre o assunto ou incerteza e falta de clareza nos resultados alcançados até o momento, o que revela a necessidade de desenvolvimento de mais estudos relacionando as marcas como indicadores. E a maior proximidade das maras com inovação, produtos, serviços, indicador de inovação e indicador econômico induz o caminho a ser seguido pelos futuros estudos.

Com a finalidade de identificar quais referências mais utilizadas nos documentos e apontar quais estudos foram mais relevantes para a construção de seus arcabouços teórico e empírico, a Figura 7 exibe a rede de cocitação entre as referências utilizadas pelos autores dos documentos selecionados com mais de uma ocorrência.

Das 247 referências utilizadas em todos os documentos, 16 delas foram citadas mais de uma vez, dentre elas destacam-se por ordem de relevânciaSchmoch (2003), Mendonça et al., (2004), Economides (1988) ${ }^{4}$ e Allegrezza e Guarda-Rauchs (1999). 


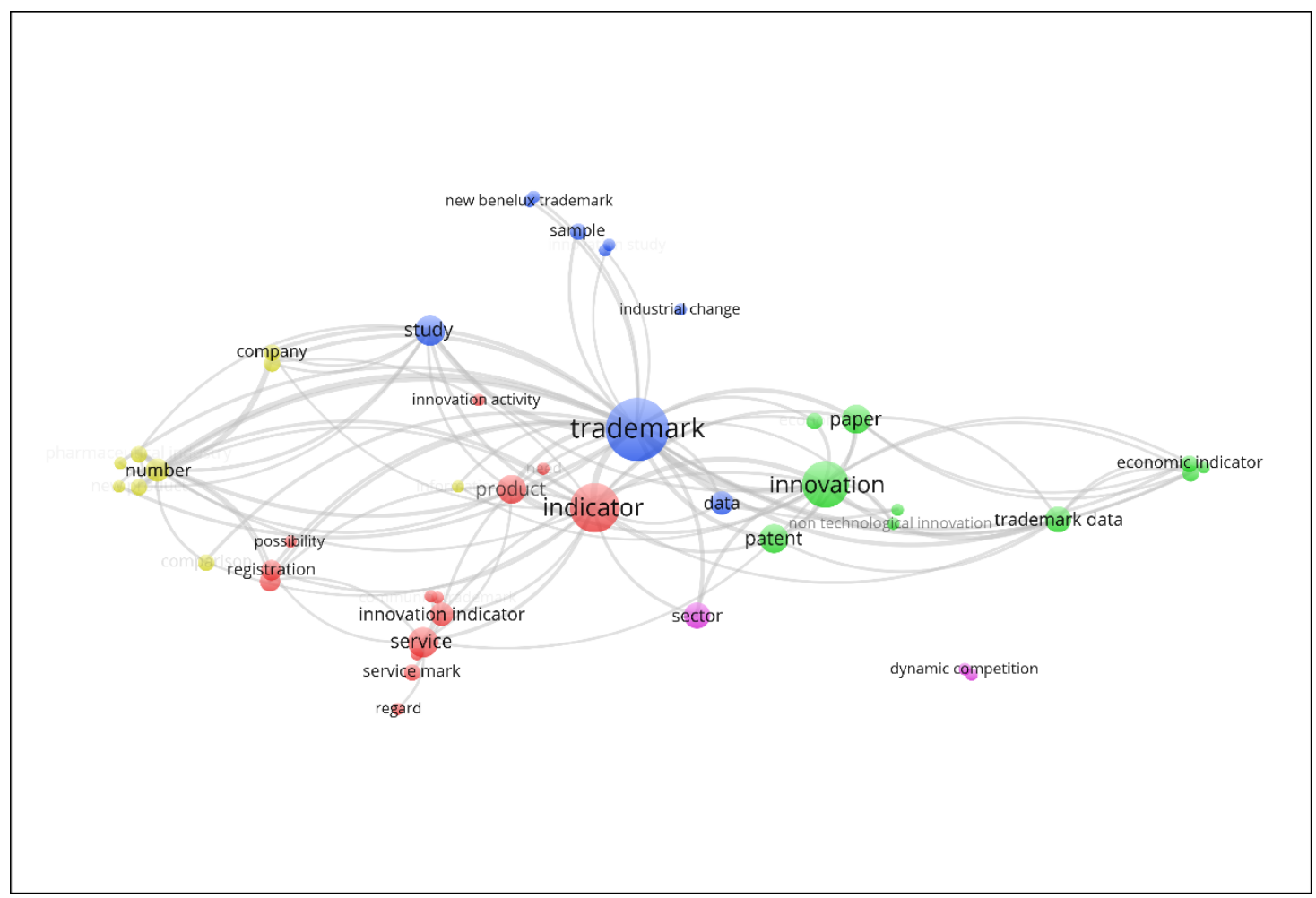

Figura 4. Rede dos temos utilizados nos títulos e resumos dos documentos.

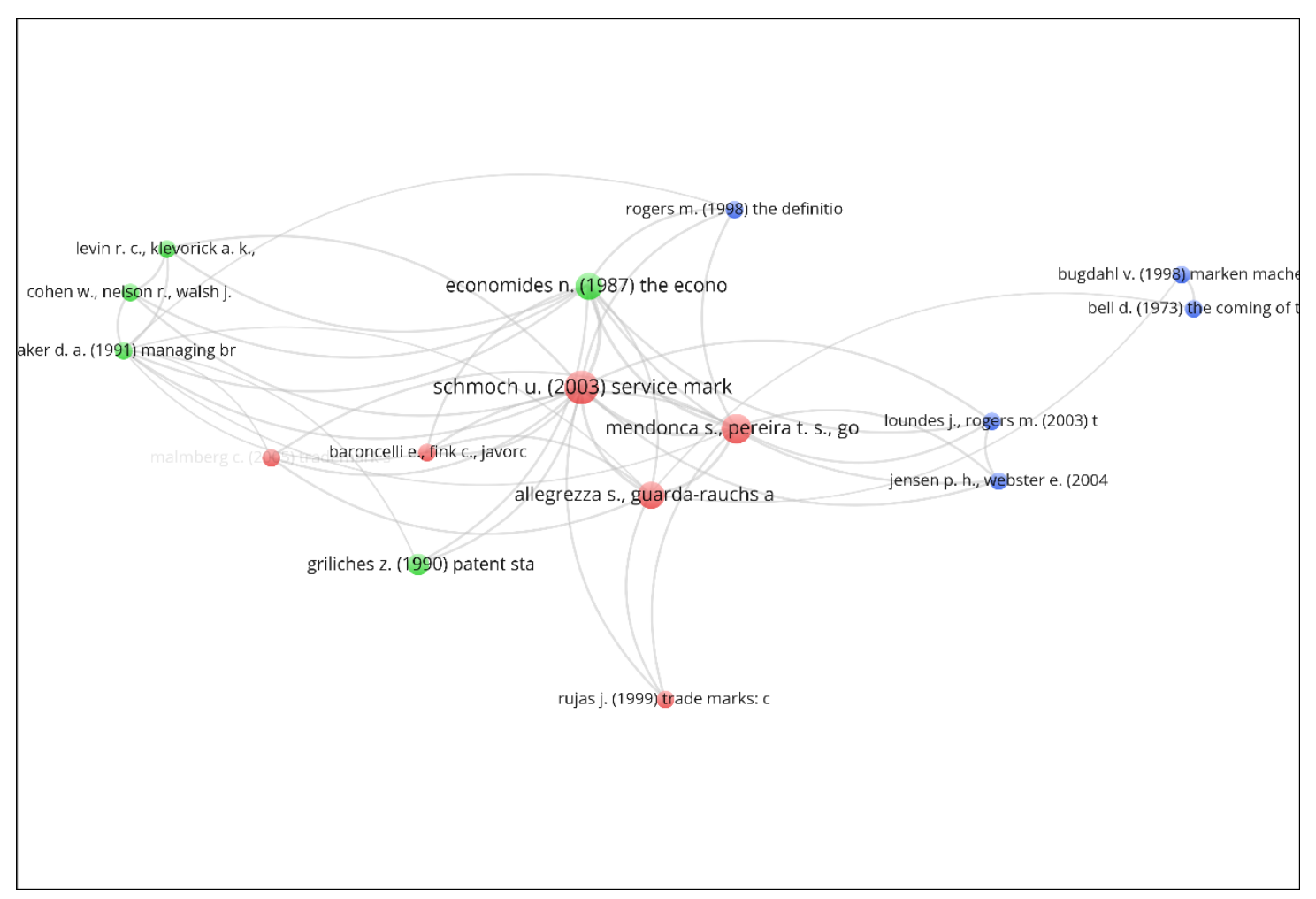

Figura 7. Cocitação de referências. 


\subsection{Análise sistêmica}

A Tabela 5 sintetiza os resultados encontrados nos documentos, especialmente em relação aos tipos de indicadores aos quais as marcas são relacionadas e que podem ser utilizadas para mensurar, em conformidade com os resultados obtidos.

Com fundamento na análise sistêmica concretizada observa-se que, não obstante a pouca quantidade de publicações que relacionam as marcas com algum tipo de indicador, existe uma quantidade significativa de aplicações que podem ser exploradas. Sobretudo em relação a diferentes tipos e graus de inovação, tipos de empresas e mercados, além da relação com a atividade econômica.

O estudo feito por Flikkema et al. (2014) baseou-se em uma amostra de 660 empresas de Benelux ${ }^{5}$ que solicitaram registro de marca no Escritório Benelux de Propriedade Intelectual (BOIP) entre janeiro de 2007 e março de 2008. 95\% das empresas que responderam o questionário são pequenas e médias empresas (com menos de 50 empregados) e, majoritariamente, pertencentes a ramos de serviços.

58 por cento dos pedidos de registro de marca analisados referem-se a pelo menos uma das seis classificações de inovação utilizadas no estudo (produto, processo, serviço, tecnológica, organizacional e de marketing). Ademais, o estudo identificou que as marcas são particularmente valiosas como medidas de inovação em serviços. No entanto, parte substancial das marcas referentes à inovação de produtos não foi combinada com patentes, o que leva a crer que também são aptas a medir este tipo de inovação.

Tabela 5. Síntese da análise sistêmica dos documentos.

\begin{tabular}{|c|c|}
\hline Referência & Tipo de indicador \\
\hline Flikkema et al. (2014) & $\begin{array}{l}\text { Indicador de inovação para pequenas e médias empresas (PMEs). } \\
\text { Inovações de produtos de base tecnológica e inovações não tecnológicas, } \\
\text { especialmente para invações em serviços e inovações em estágios } \\
\text { avançados. }\end{array}$ \\
\hline Gatrell and Ceh (2003) & $\begin{array}{l}\text { Indicador de inovação, novas atividades econômicas, prever mudanças } \\
\text { econômicas e para explorar sistemas de inovação regionais. }\end{array}$ \\
\hline Malmberg (2005) & $\begin{array}{l}\text { Indicador de inovações do tipo novo para a empresa relacionadoas a } \\
\text { novos produtos. }\end{array}$ \\
\hline Meeusen and Rayp (2000) & Indicador de competitividade internacional para países. \\
\hline Mendonça (2012) & $\begin{array}{l}\text { Medida de como um país está tendo sucesso na transição estrutural para } \\
\text { uma economia baseada no conhecimento, indicador da capacidade } \\
\text { comercial, indicador econômico de inovação e mudança industrial. }\end{array}$ \\
\hline Mendonça et al. (2004) & Indicador de atividade inovadora. \\
\hline Millot (2009) & $\begin{array}{l}\text { Indicador para medir a inovação, notadamente relacionada a produtos, } \\
\text { serviços e marketing. }\end{array}$ \\
\hline Schmoch (2003) & Marcas de serviço como indicador de inovação. \\
\hline Schmoch and Gauch (2009) & $\begin{array}{l}\text { Marcas de serviço como indicador para a análise quantitativa das } \\
\text { atividades de inovação nos serviços. }\end{array}$ \\
\hline
\end{tabular}

Fonte: Elaboração Própria

Os autores conseguiram depreender a importância dos motivos de registros de marcas relacionados à inovação, bem como uma alta referência das marcas com inovação. O estudo, por conseguinte, confirma a validade das contagens de marcas como um indicador de inovação para pequenas e médias empresas (PME).

Além disso, constatou-se que contagens de marcas permitem uma melhor medição da inovação de serviços e também de inovações em fases avançadas do processo de inovação. E os dados alcançados mostram que as 
marcas ainda fornecem informações ao medir a inovação de produtos baseadas em tecnologia e que revelam múltiplos tipos de inovação, incluindo a inovação não tecnológica.

Os resultados deste estudo foram considerados particularmente relevantes para os decisores políticos, que costumam usar principalmente as despesas de P\&D e dados de patentes como indicadores de inovação. Ao incluir as marcas registradas como um indicador de inovação, pode-se fazer mais justiça às diferenças entre as indústrias e à servitização da sociedade.

Por fim, o estudo concluiu que marcas registradas também podem desempenhar um papel na avaliação de políticas. Caso mais marcas comerciais forem registradas como consequência de uma nova política, isso pode mostrar a política funcionou.

Com o objetivo de explorar o uso de uma medida alternativa para avaliar a inovação e prever mudanças econômicas, Gatrell e Ceh (2003) analisaram dados de todos os 50 estados dos Estados Unidos de 1996 à 2000. Dados como produto estadual bruto per capita, renda pessoal per capita, total anual de pedidos de registro de marca registrada por estado, marcas registradas totais per capita, proporção de pedidos de registro de marcas concedidas, total anual de pedidos de patentes por estado, patentes concedidas per capita e proporção de pedidos de patentes concedidas foram analisados.

Os resultados sugerem que as marcas podem ser uma medida efetiva de inovação e podem ser usadas para explorar sistemas regionais de inovação. Ainda que de forma imperfeita, as marcas medem atividades que patentes não conseguem captar. Além do mais, o estudo ressalta que a pesquisa sobre marcas pode revelar-se uma área de pesquisa interessante e promissora e incentiva, ainda, futuras pesquisas e discussões da relação entre marcas e economias regionais, a geografia da produção de marca registrada e os determinantes locais da produção de marca registrada.

Malmberg (2005) investigou o uso de marcas como um indicador de inovações do tipo novas para a empresa, ou seja, novos produtos. Para tanto, selecionou algumas empresas suecas e analisou suas estatísticas de marcas na tentativa de falsificar a hipótese de que as marcas registradas refletem novos produtos. As empresas estudadas representavam dois setores da indústria principal, os setores de engenharia e farmacêutico. Dentro da engenharia selecionou-se três empresas da indústria eletromecânica e duas do setor automotivo. O setor farmacêutico foi estudado na íntegra, com as 13 empresas suecas relevantes.

O período de tempo estudado difere para as diferentes partes da investigação, mas, em geral, abrangeu-se um longo período de aproximadamente a década de 1940 até a década de 1990. Os resultados indicam que as marcas comerciais geralmente não são confiáveis como indicador de novos produtos para as empresas estudadas nas indústrias eletromecânicas e automotivas.

Já os resultados alcançados em relação a indústria farmacêutica foram diferentes. O número de marcas registradas é muito maior que o número de medicamentos recém-registrados. Não obstante, a contagem de marcas registradas na classe 5 (relativa a área farmacêutica) é cointegrada com o número de novos medicamentos, o que indica que as marcas registradas, pelo menos a longo prazo, estão refletindo inovações novas para a empresa.

Como a contagem de marcas registradas na indústria farmacêutica contém informações sobre o processo de inovação, tanto a curto quanto a longo prazo, isso sugere que as estatísticas de marcas registradas podem ser adequadas como indicador de inovação para outras indústrias com um processo de desenvolvimento menos incerto do que em produtos farmacêuticos e que registram marcas com frequência. Mas os resultados negativos para as empresas eletromecânicas e automotivas sugerem que o uso de marcas registradas como indicador de inovação deve ser feito de forma seletiva, provavelmente por base industrial.

Meeusen e Rayp (2000), ao examinar a competitividade internacional da indústria transformadora de 12 países da Organização para a Cooperação e Desenvolvimento Económico ou Econômico (OCDE), no período 19701995, utilizaram as participações nacionais nos pedidos de marcas estrangeiras nos EUA como uma variável de atividade de inovação comercial.

O estudo mostra que os países que perderam participação no mercado no período considerado também viram a sua parcela relativa das despesas de P\&D no Produto Interno Bruto (PIB) diminuir, e/ou perda de participação em patentes ou marcas registradas.

As participações nos pedidos de marca registrada nos EUA foram significativas em todas as equações de competitividade testadas, exceto para a França e a Suécia. Isso pode ser visto como uma confirmação do 
pressuposto de que as marcas realmente medem algo além de esforços de inovação e podem ser interpretados como um indicador independente de competitividade significativo.

Além do mais, para a maioria dos países da amostra as variáveis de participação em patentes e/ou a participação em marcas registradas mostraram-se estatisticamente significativas. Para alguns, até mesmo ambas as variáveis foram significativas. Pôde-se observar, ainda, a influência independente e significativa dos aspectos comerciais da inovação, medida pela participação em marcas registradas.

Mendonça et al. (2004) avaliam as possibilidades e os problemas do uso de dados de marca registrada ao analisar a introdução de produtos novos ou melhorados em mercados competitivos. Seu estudo apresenta uma aplicação empírica concreta do indicador nos 15 países da União Euripéia, mais um estudo aprofundado de uma economia européia intermediária, a de Portugal.

O estudo mostra que a evidência do caso português sugere que as empresas que tendem a usar um tipo de direito de propriedade intelectual também usam outros tipos. Isso implica que os setores de alta tecnologia, que usam mais patentes, também fazem um uso mais intensivo de marcas registradas. Os dados também mostram que as indústrias de serviços geralmente classificadas como usuários intensivos de informações são as que usam a maioria das marcas registradas.

Os autores argumentam que os dados de marcas podem servir para atuar como um indicador parcial de produção de inovações introduzidas nos mercados de bens e serviços e, portanto, podem ser usados como um padrão empírico para medir as mudanças gerais nos padrões de atividade econômica. E que isso pode ser especialmente útil para o avanço da pesquisa em estudos de inovação, dinâmica industrial e economia internacional, bem como na história econômica e comercial.

Posteriormente, aprofundando especialmente a inovação na área de telecomunicações, Mendonça (2012) explorou as novas marcas comunitárias de telecomunicações dos 15 países da União Euripéia no período 19962009. Além de realizar uma taxonomia dos países acerca de suas capacidades tecnológicas e de marketing, verificar a concentração nos serviços de telecomunicações e identificar a instabilidade do mercado nos serviços de telecomunicações na União Europeia, o estudo concluiu que as marcas são únicas, todavia ainda são uma fonte de informação subexplorada na pesquisa do comportamento da inovação e a dinâmica industrial no setor de telecomunicações.

Millot (2009) comparou diversos dados de marcas de vários países diferentes e conclui que, embora o crescimento de pedidos de registro de marcas seja aproximadamente contínuo, a atividade de marca registrada é impactada pelas flutuações gerais da atividade econômica. Além disso, além dos serviços, pedidos de registro de marca parecem ser numerosos nos campos de produtos onde a atividade de patenteamento é baixa.

Para o autor, dados de marcas registradas divulgam informações sobre dois aspectos chave da inovação que não são bem cobertos por indicadores tradicionais: inovação em marketing e inovação nos setores de serviços. Ademais, estudos empíricos têm demonstrado vínculo contagem de marcas e outros indicadores de inovação. Por exemplo, números de marcas registradas foram encontrados positivamente correlacionados com inovação, como refletido nas respostas a pesquisas de inovação, com P\&D (para certas indústrias), com patentes e com o número de lançamentos de novos produtos. Tal correlação se mostra particularmente elevada em serviços intensivos em conhecimento e em setores de alta tecnologia, como a indústria farmacêutica.

Schmoch (2003) investigou dados de pesquisa para empresas alemãs, e como resultado geral obteve uma correlação altamente significativa entre inovação e marcas. E assim considera as marcas de serviço adequadas para a construção de indicadores de inovação, na medida em que atendem condições fundamentais, tais como correlação com a inovação, o bom acesso a dados por bancos de dados eletrônicos e a possibilidade de operacionalizá-las em dimensões relevantes de desagregação.

Como áreas promissoras de estatísticas de marcas, o autor definiu a análise de serviços inteligentes de conhecimento e de serviços de acompanhamento de produtos, uma vez que ambos são relevantes para a competitividade de países avançados. E assim será possível examinar a relação entre tecnologia e serviços de forma detalhada. Em síntese, dados de marcas revelam-se uma nova e desafiadora ferramenta para apoiar uma melhor compreensão do papel dos serviços na economia atual.

Schmoch e Gauch (2009), elaboraram um conceito de como as marcas podem ser utilizadas como base quantitativa para medir a inovação nas indústrias de serviços. E a partir dele concluíram que os registros de 
marcas revelam bem a tendência da atividade econômica e que, de modo geral, as marcas revelam-se indicadores adequados para a análise quantitativa das atividades de inovação nos serviços.

Bem como, que as estatísticas de marcas têm um enorme potencial de análise da inovação, pois, na situação atual, as marcas são o único enfoque para uma descrição estatística da produção de inovação em serviços baseados no conhecimento que são disponíveis facilmente e a tempo e já foram testados no nível micro.

\section{Conclusões}

O presente estudo teve como objetivo investigar, apresentar e discutir os conceitos, contextos e aplicações referentes ao uso de marcas como indicadores já explorados na literatura. Além de evidenciar o que pode ser inferido a partir de pedidos de registro de marcas, e quais conclusões foram obtidas com base nas publicações encontradas.

Para tanto, técnicas de revisão sistemática da literatura foram empregadas e as buscas se deram em várias bases de publicações, com o intuito de cobrir um grande leque de publicações disponíveis.

A análise bibliométrica realizada encontrou algumas particularidades inconvenientes acerca dos documentos selecionados, como a pouca quantidade de publicações abordando o assunto, a tendência apontando a não evolução no número anual de publicações ou que as mesmas virão a ocorrer com menor frequência. E presença de publicações em caráter inicial ou carentes de avaliação editorial (os denominados Working Papaers).

Contraditoriamente, outros documentos foram publicados em periódicos com alto fator de impacto e possuem notável incidência de citações. O que, em contrapartida, manifesta a relevância e o potencial que possui a pesquisa envolvendo a temática abordada.

$\mathrm{Na}$ análise de rede (network) observou-se pouca relação entre os autores dos documentos entre si e a ocorrência de fato semelhante entre suas respectivas instituições, além da grande distância existente entre eles. Outrossim, foi visto que o fator preponderante na para o número de citações é o fator de impacto do periódico no qual o documento foi publicado e não seu ano de publicação.

No tocante aos termos que se destacaram nos títulos e resumos dos documentos, salvo os previamente selecionados por ocasião da busca, os termos innovation, product, service, trademark dada, innovation indicator e economic indicator se sobressaíram. Demonstrando como as marcas têm sido estudadas como indicadores.

Dentre as referências utilizadas em todos os documentos, destacam-se por ordem de relevância Schmoch (2003), Mendonça et al., (2004), Economides (1988) e Allegrezza e Guarda-Rauchs (1999). Assim, podemos considerá-las as mais relevantes para a construção dos arcabouços teórico e empírico dos documentos estudados. E, portanto, ponto de partida obrigatório para quem deseja prosseguir no aprofundamento do assunto.

Após análise sistêmica dos documentos recuperados pela pesquisa sistematizada alguns pontos comuns foram constatados em quase a totalidade das publicações. Em geral, os documentos ressaltam grande relevância das marcas para as empresas, pouco desenvolvimento de pesquisas na área, necessidade de avanço e aprofundamento das abordagens desenvolvidas, grande disponibilidade de dados para futuras pesquisas, entre outros.

Em relação aos tipos de indicadores aos quais as marcas são relacionadas e para que podem ser utilizadas, os documentos selecionados apresentaram uma ampla gama de possibilidades, como indicador de novas atividades econômicas, para prever mudanças econômicas, explorar sistemas de inovação regionais, indicador de competitividade internacional, porém, com especial atenção para seu uso como indicador de inovação, o que ocorreu com maior frequência.

Como indicador de inovação, destacaram-se os usos notadamente para PMEs, produtos de base tecnológica, inovações não tecnológicas, invasões em serviços, inovações em estágios avançados, inovações do tipo novo para a empresa relacionadas a novos produtos, atividade inovadora, para medir a inovação relacionada a produtos, para medir a inovação relacionada a marketing e para a análise quantitativa das atividades de inovação nos serviços.

Diante de tantas possibilidades relatadas ressalta-se a importância dos, ainda não tão explorados, dados de marcas para os mais variados usos. Particularmente quando relacionadas a serviços, uma vez que dados 
recentes da OCDE mostram que os serviços representam mais de 70\% do PIB na maioria dos países da OCDE. Além disso, aumentos notáveis no conteúdo dos serviços do setor empresarial desde 1995 são evidentes em quase todos os setores de manufatura (OECD, 2015), o que corrobora com o fenômeno da servitização das economias aboradada por Flikkema et al. (2014).

\section{Referências}

ALBUQUERQUE, V. V. C. DE. A rose by any other name: Conformações do direito de marca no mercado farmacêutico. Revista de informação legislativa, v. 52, n. 207, p. 123-141, 2015

ALLEGREZZA, S.; GUARDA-RAUCHS, A. The determinants of trademarks deposits: An econometric investigation. Economie appliquée, v. 52, n. 2, p. 51-68, 1999.

BORNMANN, L.; DANIEL, H. What do we know about the $\mathrm{h}$ index? Journal of the American Society for Information Science and technology, v. 58, n. 9, p. 1381-1385, 2007.

BRAUN, T.; GLÄNZEL, W.; SCHUBERT, A. A Hirsch-type index for journals. Scientometrics, v. 69, n. 1, p. 169-173, 2006.

BRERETON, P. et al. Lessons from applying the systematic literature review process within the software engineering domain. Journal of Systems and Software, v. 80, n. 4, p. 571-583, 2007.

BROWN, E. J.; NAGY, P. F. That's not fair! Clarifying copyright and trademark fair use for business managers. Business Horizons, v. 58, n. 1, p. 17-24, 2015.

ECONOMIDES, N. S. The economics of trademarks. The Trademark Reporter, v. 78, n. 4, p. 523-539, 1988.

FALAGAS, M. E. et al. Comparison of SCImago journal rank indicator with journal impact factor. The FASEB journal, v. 22, n. 8, p. 2623-2628, 2008

FLIKKEMA, M.; DE MAN, A.-P.; CASTALDI, C. Are Trademark Counts a Valid Indicator of Innovation? Results of an In-Depth Study of New Benelux Trademarks Filed by SMEs. Industry and Innovation, v. 21, n. 4, p. 310-331, 2014.

GARCÍA, G. R. ¡No me dejes sin marca,¡ No me dejes sin marca, Pezweón!: consideraciones legales y económicas a favor de la abolición de las cláusulas de moralidad en el derecho de marcas. Ius Et Veritas, v. 21, n. 42, p. 336-345, 2015.

GATRELL, J. D.; CEH, S. L. B. Trademark Data as Economic Indicator: The United States, 1996-2000. The Great Lakes Geographer, v. 10, n. 1, p. 46-56, 2003

HIGGINS, J. P.; GREEN, S. (EDS.). Cochrane Handbook for Systematic Reviews of Interventions Version 5.1.0 [updated March 2011]. The Cochrane Collaboration, 2011. Disponível em: www.handbook.cochrane.org.

HUANG, M. H.; CATHY LIN, W. Y. The influence of journal self-citations on journal impact factor and immediacy index. Online Information Review, v. 36, n. 5, p. 639-654, 2012.

KITCHENHAM, Barbara. Procedures for performing systematic reviews. Keele University, v. 33, n. 2004, p. 1-26, 2004.

MALMBERG, Claes. Trademarks statistics as innovation indicator? - A micro study. CIRCLE Electronic Working Paper Series, No. 17, vol. 2005, CIRCLE, Lund University, Lund, 2005.

MARQUES, R. L. P.; MAGALHÃES, R. A. Contribuições da filosofia do Direito para a aceitação de marcas sonoras no direito brasileiro. Revista Jurídica Cesumar-Mestrado, v. 9, n. 1, p. 57-69, 2009.

MEEUSEN, W.; RAYP, G. Patents and trademarks as indicators of international competitiveness: the VAR versus the hysteresis approach. In: BUIGUES, P. (Ed.). . Competitiveness and the value of intangible assets. Cheltenham: Edward Elgar, 2000. p. 273-296.

MENDONCCA, S. Trademarks as a telecommunications indicator for industrial analysis and policy. In: HADJIANTONIS, A. M. STILLER, B. (Eds.). . Telecommunication Economics. Berlin, Heidelberg: Springer Berlin Heidelberg, 2012. p. 33-41.

MENDONÇA, S.; PEREIRA, T. S.; GODINHO, M. M. Trademarks as an indicator of innovation and industrial change. Research Policy, v. 33, n. 9, p. 1385-1404, 2004.

MILLOT, V. Trademarks as an Indicator of Product and Marketing Innovations: OECD Science, Technology and Industry Working Papers, 2009/06. Paris: 2009.

OECD. OECD Science, Technology and Industry Scoreboard 2015: Innovation for growth and society. Paris: OECD Publishing, 2015. 
ONO, S. Overview of Japanese Trademark Law. 2nd. ed. Tokyo: Yuhikaku, 1999.

PETTICREW, M.; ROBERTS, H. Systematic reviews in the social sciences: A practical guide. Oxford: John Wiley \& Sons, 2008

PLAZA, A. T. Introducción al régimen jurídico de la marca notoria y de la marca renombrada en la nueva ley española de marcas. Ius Et Veritas, v. 15, n. 31, p. 30-38, 2015.

PRINCETON UNIVERSITY. What are working papers? <https://www. princeton.edu/ pswpc/about/about.html>. Acesso em: 1 fev. 2017.

PRODANOV, C. C.; FREITAS, E. C. DE. Metodologia do trabalho científico: métodos e técnicas da pesquisa e do trabalho acadêmico. 2. ed. Novo Hamburgo: Feevale, 2013.

SANTOS, R. N. M. DOS. Produção científica: por que medir? o que medir? Revista Digital de Biblioteconomia e Ciência da Informação, v. 1, n. 1, p. 22-38, 2003.

SCHMOCH, U. Service marks as novel innovation indicator. Research Evaluation, v. 12, n. 2, p. 149-156, 2003.

SCHMOCH, U.; GAUCH, S. Service marks as indicators for innovation in knowledge-based services. Research Evaluation, v. 18, n. 4, p. 323-335, 2009.

VAN ECK, N. J.; WALTMAN, L. Software survey: VOSviewer, a computer program for bibliometric mapping. Scientometrics, v. 84, n. 2 , p. $523-538,2010$.

VICTOR, L. Systematic Reviewing in the Social Sciences: Outcomes and Explanation. Enquire, v. 1, n. 1, p. 1-12, 2008.

WIPO. WIPO Intellectual Property Handbook: Policy, Law and Use. 2. ed. Geneva: WIPO, 2008.

WTO. Agreement on Trade-related Aspects of Intellectual Property Rights. WTO, 1994. 


\title{
Dados dos autores
}

\section{Adonis Reis de Medeiros Filho}

Mestre e Doutorando em Ciência da Propriedade Intelectual na Universidade Federal de Sergipe.

adonisrmf@gmail.com

Suzana Leitão Russo

Professora Adjunta do Programa de Pós-graduação em Ciência da Propriedade Intelectual da Universidade Federal de Sergipe (PPGPI/USF).

suzana.ufs@hotmail.com

Recebido - Received: 2017-06-13

Aceitado - Accepted: 2018-01-11

\begin{abstract}
1 Incluindo todo o conteúdo disponível em cada banco de dados, como todos os tipos de publicações e bancos de dados disponíveis em cada uma delas.

2 O campo "Título" foi escolhido porque as pesquisas envolvendo o campo "Título e Resumo" apresentaram resultados com mais de 70.000 (setenta mil) documentos, o que tornaria a pesquisa inviável.

${ }^{3}$ De acordo com a Universidade de Princeton (2005), Working Papers são versões pré-publicação de artigos, capítulos de livros ou revisões. São pesquisas em andamento, em submissão, ou futuramente em outros lugares. Working Papers não passam por processo de avaliação e podem estar em vários estágios de conclusão.

${ }^{4}$ Apesar de ser referenciado pelos documentos com data de 1987 o referido artigo é de 1988, conforme consta na capa do volume no qual foi publicado (volume 78), consoante base de dados HeinOnline (http://heinonline.org/HOL/LandingPage?handle=hein.journals/thetmr78\&div=1\&src=home).
\end{abstract}

${ }^{5}$ Organização econômica que compreende a Bélgica, Holanda e Luxemburgo.

\section{(c) EY}

This work is licensed under a Creative Commons Attribution 4.0

United States License.

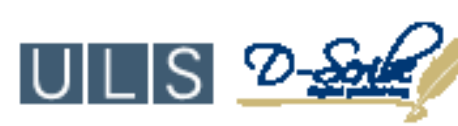

This journal is published by the University Library System of the University of Pittsburgh as part of its D-Scribe Digital Publishing Program and is cosponsored by the University of Pittsburgh Press. 